\title{
La prensa escrita y su contribución a la lectura crítica y la escritura argumentada
}

\section{The written press and its contribution to critical reading and argued writing}

\author{
Rudy Doria Correa \\ Magister en Educación \\ Universidad de Córdoba \\ Colombia \\ doriarudy@hotmail.com
}

\author{
Benjamín Calle González \\ Magister en Educación \\ Institución Educativa Germán Gómez Peláez \\ Colombia \\ benjamíncalle_07@hotmail.com
}

\author{
Juan Pérez Solipá \\ Lic. En Literatura y Lengua Castellana \\ Universidad de Córdoba \\ Colombia \\ juperz00@gmail.com
}

\section{Resumen}

Palabras claves:

\section{Secuencia}

didáctica, Enfoque

comunicativo-

discursivo, Lectura

crítica, Escritura

argumentada.
Este artículo muestra los resultados de un proyecto de investigación realizado en la Institución Educativa Germán Gómez Peláez, de Puerto Libertador - Córdoba, con el objetivo de establecer la contribución del trabajo con la prensa escrita, desde un enfoque comunicativo del lenguaje, en el desarrollo de la lectura crítica y escritura argumentada de los estudiantes de grado $10^{\circ}$. Teóricamente se sustenta en la concepción comunicativa y discursiva del lenguaje, y se apoya en los postulados de autores como María Cristina Martínez, Daniel Casanny, Carlos Lomas, entre otros. Se asumió una metodología de carácter cualitativo interpretativo que comprendió tres fases: exploratoria, con el objetivo de analizar el enfoque del lenguaje y las estrategias didácticas que aplican los profesores en la enseñanza de la lectura y la escritura, mediante la aplicación de una entrevista semiestructurada a 4 docentes de lenguaje; ejecución, que comprendió el diseño y aplicación de un sistema de secuencias didácticas, desde la perspectiva comunicativa del lenguaje, para el desarrollo de la lectura crítica y la escritura argumentada a 30 estudiantes de grado $10^{\circ}$; valoración, se realizaron grupos de discusión con el fin de evaluar los resultados de la aplicación del sistema de secuencias didácticas, teniendo en cuenta las percepciones de los estudiantes sobre sus prácticas de comprensión y producción escrita. Los resultados de la aplicación de las secuencias didácticas, permitió a los estudiantes leer e identificar las intenciones y propósitos comunicativos de los textos de opinión, sus enunciados e inclinaciones ideológicas y las intenciones de manipulación y manejo del poder. Del mismo modo, se evidenciaron avances en su capacidad de escribir columnas de opinión teniendo en cuenta los elementos micro, macro y superestructurales de los textos, al tiempo que mejoraron sus estrategias de argumentación.

Recepción: Mayo 09 de 2020 | Aceptación: Junio 12 de 2020 | Publicación: Junio 30 de 2020 


\section{Abstract}

Keywords:

Didactic sequence, Communicativediscursive approach, Critical reading, Argumented writing.
This article shows the results of a research project carried out in the educational institution German Gomez Peláez, in Puerto Libertador Cordoba, with the objective of establishing the contribution of the work with the written press, from a communicative approach to language, in the development of critical reading and argumentative writing of 10th grade students. Theoretically it is based on the communicative and discursive conception of language, and it is based on the postulates of authors such as Maria Cristina Martinez, Daniel Casanny, Carlos Lomas, among others. An interpretative qualitative methodology was adopted and comprised three phases: exploratory, with the objective of analyzing the language approach and the didactic strategies applied by teachers in the teaching of reading and writing, through the application of a semi-structured interview to 4 language teachers; Execution, which included the design and application of a system of didactic sequences, from the communicative perspective of language, for the development of critical reading and argumentative writing of 30 10th grade students; Evaluation, discussion groups were held in order to evaluate the results of the application of the system of didactic sequences taking into account the perceptions of students about their practices of comprehension and written production. The results of the application of didactic sequences, allowed the students to identify the communicative intentions and purposes of opinion texts, their statements and ideological inclinations, and the intentions of manipulation and management of power. In the same way, there were advances in his ability to write opinion columns taking into account the micro, macro and superstructural elements of the texts, as well as argumentation strategies.

Received: May 09, 2020 | Accepted: June 12, 2020 | Published: June 30, 2020 


\section{Introducción}

Superar las dificultades en la comprensión lectora y la producción escrita con las que llegan los estudiantes colombianos a la media académica, es uno de los mayores desafíos que enfrentan los maestros de lenguaje de nuestras escuelas actualmente. El Segundo Estudio Regional Comparativo y Explicativo de SERCE. Aportes para la enseñanza de la lectura (Atorresi y Colaboradores, 2012), da cuenta de cómo estas falencias empiezan en primaria y trascienden a la secundaria. El estudio se aplicó a estudiantes de $3^{\circ}$ y $6^{\circ}$ grados en 16 países de América Latina, evaluando la comprensión lectora en textos narrativos, descriptivos, explicativos, instructivos y argumentativos. En grado sexto, por ejemplo, Colombia obtuvo de 12 indicadores evaluados, sólo dos en buen nivel: uno en A (Alto) en la categoría de Cuento, y otro en SA (Significativamente Alto) en Fábula; el resto, aparecen es su mayoría en nivel Medio (M) y otros en Bajo (B). La categoría de Argumentación, en el indicador Cartas de Lectores, aparece en M (Medio); la valoración correspondiente, en términos porcentuales para este mismo indicador, en sexto grado, es de $48.81 \%$. Entre todos los países evaluados, la Argumentación (47.71) y la Crónica (46.72) están entre los cuatro puntajes más bajos del estudio.

Si se relacionan estos datos con los ya conocidos de las Pruebas ICFES, Saber y PISA, que son reiterativos sobre bajos niveles de lectura en estudiantes de $9^{\circ}$ y $11^{\circ}$ grados, se conjetura que los progresos no son nada alentadores en habilidades comunicativas tan fundamentales como son leer comprensivamente y escribir de manera cohesiva y coherente. Ahora, si se habla de lectura crítica y de escritura argumentada, tal como lo solicita actualmente el MEN, y de acuerdo a cómo se plantea desde las tendencias actuales de enseñanza y aprendizaje del lenguaje, se entiende que las escuelas y los maestros tendrán que hacer esfuerzos cada vez mayores para la implementación de nuevas maneras de aprender y desarrollar estas habilidades por parte de los estudiantes.

Frente a esta problemática han surgido variadas alternativas de trabajo docente, distintas al enfoque tradicional de enseñanza basada en contenidos, entre ellas la elaboración de secuencias didácticas (ampliamente recomendadas en el Programa Todos a Aprender -PTAMEN (2012), en Colombia), y en algunos casos la educación por proyectos (tal como lo sugieren los Lineamientos Curriculares de Lengua Castellana en 1998), los cuales se ofrecen como medios para organizar mejores situaciones del aprendizaje que favorezcan la participación dinámica de los estudiantes, poniéndose a tono con el debate didáctico contemporáneo centrado en el aprendizaje, antes que en la enseñanza, que enfatiza en la responsabilidad pedagógica del docente para proponer a sus alumnos actividades secuenciadas, encaminadas a establecer un clima democrático y participativo en el que, la actividad de leer y escribir cobre mayor sentido e importancia (Díaz-Barriga, 2013).

Estas alternativas de trabajo sociocultural privilegian el intercambio de ideas y la construcción del conocimiento compartido mediante el aprendizaje recíproco en el aula de clases. Es decir, estas prácticas le permiten al docente y al estudiante asumir sus roles y participar de una interacción constante, que posibilita mantener un vínculo o una reciprocidad 
de saberes dentro y fuera del aula, contribuyendo así, al desarrollo de las habilidades comunicativas de los estudiantes (hablar, escuchar, leer y escribir), pues estas se desarrollan mediante procesos interactivos en los que "el estudiante participe y reflexione sobre el uso de la lengua en diferentes situaciones orales y escritas, las cuales le permiten comprender y producir diferentes estilos discursivos: narración, descripción, explicación, argumentación, diálogo"(Calsamiglia y Tusón, 1999, p. 24).

La perspectiva comunicativa y discursiva del lenguaje constituye, entonces, un pilar importante para la construcción del sujeto en su integridad, pues éste se capacita para comunicarse socialmente, dar significado y finalmente dar sentido a todo cuanto lo rodea. "Los enfoques comunicativos de la educación lingüística (...) insisten en este sentido en la idea de orientar la enseñanza del lenguaje hacia el aprendizaje de los conocimientos, de las habilidades, de las estrategias y de las actitudes que contribuyen a la adquisición y al desarrollo de la competencia comunicativa de las personas" (Lomas, 2018, p. 12). Esta postura, apoyada en los desarrollos de disciplinas como la pragmática, el análisis del discurso, la sociolingüística y la lingüística del texto, ha superado la enseñanza tradicional de la lengua basada en el estudio del sistema abstracto e inmanente de la lengua.

Este trabajo se realizó desde esa perspectiva comunicativa, la cual aporta elementos teórico-prácticos para discernir las intenciones comunicativas de los textos de opinión publicados en la prensa escrita, con referencia a los aspectos de carácter discursivo y pragmático que dan cuenta de los mensajes explícitos e implícitos en los argumentos que soportar los artículos y columnas de opinión de periódicos y revistas de carácter regional y nacional. Los ejercicios prácticos se realizaron mediante la aplicación de secuencias didácticas referidas tanto a la comprensión como a la producción de textos con estudiantes de grado décimo, en un período aproximado de dos años entre 2018 y 2019 . Mediante este proceso se logró aproximar a los estudiantes a la comprensión crítica mediante el desentrañamiento de significados y sentido de los textos: las voces implícitas, los enunciadores y enunciatarios de los discursos, el tono con que se expresen los argumentos, el manejo de recursos lingüísticos como los conectores y el léxico, lo mismo que las fuentes en las que se apoyan los articulistas y los medios para emitir sus contenidos. Ese desentrañamiento de la comprensión textual sirvió como base para armar la estructura de producción de los escritos.

\section{Referentes teóricos}

\section{La lectura crítica y la escritura argumentada mediadas por la prensa escrita}

La lectura crítica y la escritura argumentada corresponden al mismo correlato. Leer críticamente exige al lector cómo está estructurado el texto en su micro y macro estructura, es decir, cómo se ha dispuesto el tejido textual (cohesión local) para lograr el significado global (coherencia). En ese proceso, el lector/escritor debe activar su competencia lingüística 
estratégica, aquella que le permite discernir sobre la función de las palabras y los enunciados en el logro de la intención comunicativa de los textos en relación con el contexto, sea cuando lee o cuando escribe.

Actualmente, la formación de lectores críticos se constituye en un objetivo primordial y en una responsabilidad social de las instituciones educativas colombianas. Esto se debe a múltiples necesidades y razones de carácter pedagógico, lingüístico, social, cultural y político que son propias una sociedad global como la actual, permeada por infinidad de medios de comunicación y de múltiples informaciones a través de las cuales se emiten diversos mensajes y contenidos que, según la capacidad del lector son comprendidos, al tiempo que favorecen los aprendizajes y la construcción de conocimientos; pero también, ante la falta de competencia del lector, pueden vulnerar sus formas de ser y de pensar al no poder discernir, con sentido crítico, la intención comunicativa de los textos y enunciados que circulan en la realidad social existente.

La lectura crítica es una competencia de carácter comunicativo, discursivo e ideológico que los estudiantes deberían adquirir en su proceso formativo, no sólo porque dicha habilidad sea evaluada en las Pruebas Saber (la escuela no debe enseñar en función de las pruebas estandarizadas), sino porque es fundamental para la vida. Por ello, en una sociedad globalizada y en constante cambio como la actual, es necesario que los estudiantes cuenten con una capacidad de comprensión lectora avanzada. Capacidad, que no sólo les permitirá analizar y comprender los textos que circulan a través de diversos medios, sino, asumir posturas críticas que se traduzcan en propuestas y acciones transformadoras orientadas a lograr una vida mejor, en contextos de justicia y equidad.

Ahora bien, a la hora de escribir se debe tener en cuenta el género discursivo y todo lo concerniente a la situación de enunciación, como lo plantea Martínez (2001), es decir, determinar, a fuerza de la razón, qué rol se asume como enunciador, cuál es la intención comunicativa, los puntos de vista a defender, los posibles enunciatarios, entre otros aspectos que son de vital importancia antes de definir, construir y escribir un discurso argumentativo. Y señala además, a manera de cuestionamiento, que "existe en la escuela un proceso evasivo en relación con la argumentación, y no sólo en primaria, sino en secundaria y aún en la universidad, debido al carácter polémico que el aprendizaje de la argumentación puede generar" (Martínez, 2001, pág. 165).

Por eso, en este trabajo se toma como objeto de estudio la prensa escrita, en tanto que brinda la posibilidad de abordar las dos habilidades: lectura crítica y escritura argumentada, habida cuenta que el propósito de los medios periodísticos, de acuerdo con su propia percepción o consideración ha sido estructurarse para convertirse en una especie de conciencia colectiva, capaz no sólo de conocer los sucesos sobre el acontecer cotidiano a nivel local, nacional e internacional, sino de valorarlos, convirtiéndose así en fuerza o poder con capacidad de penetración en la dinámica cultural de la gente, y cuya información puede ser desentrañada sólo por un lector voraz, activo y especializado. 


\section{Propósito pedagógico y disciplinar de las secuencias didácticas}

En este estudio se asume la secuencia didáctica como un ciclo de aprendizaje orientado a la realización de una tarea mediante actividades articuladas en un determinado período, con el propósito de lograr unos objetivos de aprendizaje concretos, con base en una pedagogía del acuerdo. Es una herramienta integrada por una serie de actividades de complejidad progresiva, producto de un acto de configuración, entendido como la manera particular que despliega el docente en favor de los procesos de construcción de conocimientos (Litwin, 1997). De esta manera, la secuencia didáctica se integra en un proyecto de aula en el que docentes y estudiantes trabajan de manera consensuada, teniendo como punto de partida la escritura inicial de un texto por parte del estudiante, y sobre ese texto se avanza mediante talleres progresivos de construcción textual que terminan en un producto final, previamente concertado. En el caso de este trabajo, se siguió el siguiente esquema planteado por Dolz y Schneuwly (1998, p. 93).

Ilustración 1. Esquema de una secuencia didáctica.

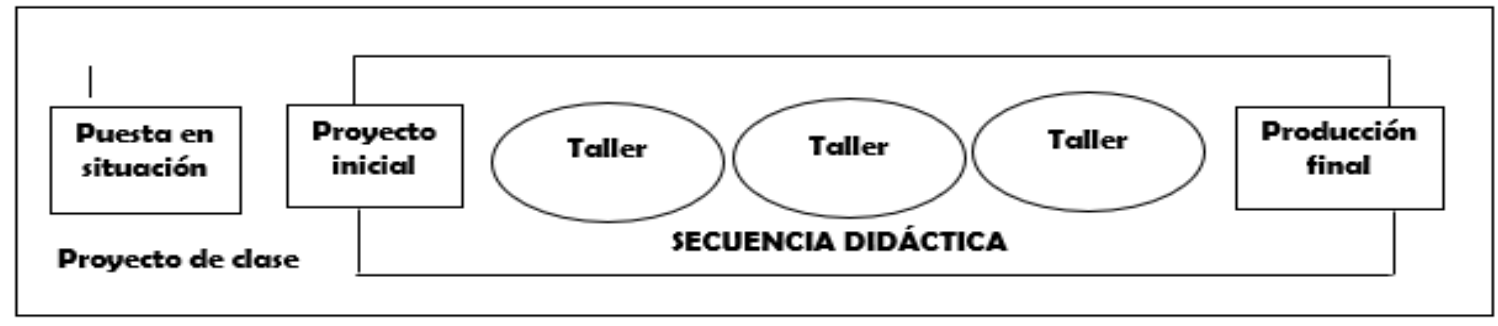

Fuente: Dolz y Schneuwly (1998).

La implementación de secuencias didácticas para la enseñanza de la lectura crítica y la escritura argumentada, desde un enfoque comunicativo y discursivo, implica un mayor compromiso de los docentes de lenguaje, porque supone asumir una postura pedagógica emancipadora, lo mismo que una posición ideológica y política liberadora, desde la cual se propicien espacios de diálogo que permitan formar ciudadanos capaces de dar significado y sentido a los actos comunicativos en los que les toque participar como sujetos críticos y democráticos, en el entendido de que "una persona crítica es la que mantiene una actitud beligerante en la consecución de sus propósitos personales, a través de la lectura y la escritura, pero también la que participa de modo constructivo en el desarrollo de una comunidad plural, respetuosa y progresista" (Cassany, 2003, p. 114).

Por eso, en este trabajo se asume, pedagógicamente, la secuencia didáctica como una estrategia interactiva e integradora que permite organizar, de manera sistemática y consensuada, un plan de trabajo con los estudiantes orientado, en este caso, al desarrollo de la lectura crítica y la escritura argumentada, haciendo uso de textos periodísticos como el artículo y la columna de opinión, dado su carácter argumentativo, su variedad temática y su profundidad crítica, cuyos propósitos fundamentales se centran en la defensa de uno o varios puntos de vista a partir de argumentos claros y contundentes. 


\section{Metodología}

Se asumió una metodología de carácter cualitativo interpretativo. En el enfoque cualitativo se "estudia la realidad en su contexto natural, tal y como sucede, sacando e interpretando fenómenos de acuerdo con las personas implicadas" (Blasco y Pérez, 2007, p.17), y a partir de esa interpretación se pueden proponer transformaciones y mejoras de los problemas que afectan a una población o grupo. El estudio comprendió tres fases:

Fase exploratoria: tuvo como propósito analizar el enfoque del lenguaje y las estrategias didácticas que aplican los profesores en la enseñanza de la lectura y la escritura. La información correspondiente a esta fase se acopió de la aplicación de una entrevista semiestructurada (Fontana y Frey, citados por Vargas, 2012) aplicada a 4 docentes de lenguaje de bachillerato, seleccionados de manera intencional, es decir, estos respondieron voluntariamente al llamado a participar en el proyecto por parte de los investigadores.

Fase de ejecución: se centró en el diseño y aplicación de un ciclo de secuencias didácticas, desde la perspectiva comunicativa del lenguaje, para el desarrollo de la lectura crítica y la escritura argumentada, en un grupo de 30 estudiantes de grado $10^{\circ}$, los cuales fueron seleccionados por conveniencia, dado que es un grupo con el cual trabajaba uno de los investigadores en su momento, de tal forma que participaron de manera consensuada con su profesor de lenguaje en la realización de las secuencias didácticas. A continuación, se ilustra el orden de actividades de dichas secuencias.

Tabla 1. Orden de las actividades dentro de las secuencias didácticas.

\begin{tabular}{|c|c|}
\hline $\begin{array}{l}\text { El orden de aplicación de las actividades de la } \\
\text { secuencia para la lectura crítica }\end{array}$ & $\begin{array}{l}\text { El orden de aplicación de las actividades de la } \\
\text { secuencia para la escritura argumentada }\end{array}$ \\
\hline $\begin{array}{l}\text { Primer momento } \\
\text { SESIÓN 1: Presentación y socialización de los propósitos de } \\
\text { la secuencia didáctica. } \\
\text { SESIÓN 2: Estudio de los medios de comunicación y su } \\
\text { función } \\
\text { SESIÓN 3: Reconocimiento de distintos medios de } \\
\text { comunicación } \\
\text { SESIÓN 4: Seleccionando información de prensa escrita } \\
\text { Segundo momento } \\
\text { SESIÓN 5: Estudio de la tesis, los argumentos y la } \\
\text { conclusión de texto de opinión: visualización } \\
\text { SESIÓN 6: Ejercicio mediante el cual el lector hace el } \\
\text { discernimiento a lo escrito en la columna de opinión y } \\
\text { asume su posición frente a los columnistas de los medios } \\
\text { de comunicación: prensa y revista. } \\
\text { SESIÓN 7: Confrontación de información entre distintos } \\
\text { textos de opinión en diferentes medios: prensa y revista. } \\
\text { SESIÓN 8: Análisis y posición del lector para el debate } \\
\text { grupal después de la lectura de varios textos de opinión } \\
\text { SESIÓN 9: Socialización de actividades de compresión: } \\
\text { debates } \\
\text { SESIÓN 10: Socialización institucional del trabajo realizado }\end{array}$ & $\begin{array}{l}\text { SESIÓN 1: Presentación y socialización de los propósitos de } \\
\text { la secuencia didáctica } \\
\text { SESIÓN 2: Seleccionando un tema de escritura } \\
\text { SESIÓN 3: Planeación de trabajo grupal: indagación de } \\
\text { fuentes } \\
\text { SESIÓN 4: Elaboración del plan textual para la escritura } \\
\text { SESIÓN 5: Redacción del primer borrador del artículo: } \\
\text { valoración } \\
\text { SESIÓN 6: Redacción del segundo borrador del artículo: } \\
\text { ajustes } \\
\text { SESIÓN 7: Reescritura grupal de artículos del texto de } \\
\text { opinión } \\
\text { SESIÓN 8: Lectura y valoración de artículos de opinión } \\
\text { SESIÓN 9: Relectura e intercambio de artículos en formato } \\
\text { digital } \\
\text { SESIÓN 10: Socialización del trabajo realizado y valoración } \\
\text { final }\end{array}$ \\
\hline
\end{tabular}

Fuente: Los autores 
Fase de valoración: realización de grupos de discusión con el fin de evaluar los resultados de aplicación del sistema de secuencias didácticas teniendo en cuenta las percepciones de los estudiantes sobre sus prácticas de comprensión y producción escrita.

\section{Resultados}

El análisis de los resultados de cada fase expuesta en la metodología se abordó de manera colectiva, es decir, con los maestros participantes se socializaron los datos de la entrevista y con los estudiantes se establecieron acuerdos para realizar las secuencias didácticas y su participación en los grupos de discusión para evaluar los resultados de los aprendizajes. Así, el carácter cualitativo e interpretativo de la investigación se cumplió en el transcurso de esta. Producto de este proceso, se emiten los resultados de acuerdo con tres categorías, coherentes con los objetivos específicos del estudio: enfoque del lenguaje de los maestros para la enseñanza de la lectura y la escritura; secuencias didácticas en lectura crítica y la escritura argumentada; y finalmente, valoraciones y aprendizajes de los participantes en la experiencia.

\section{Enfoque del lenguaje de los maestros y enseñanza de la lectura y la escritura}

Esta categoría hace referencia a la postura conceptual del lenguaje que se refleja en las prácticas de aula (estrategias didácticas) de los docentes de lenguaje. En ella se analiza si esas posturas de los maestros están relacionadas con algún paradigma de enseñanza del lenguaje, sea formal (estructuralista) o comunicativo (textual discursivo), entendiendo que el enfoque lingüístico que asuma el maestro, definirá el tipo de prácticas de enseñanza que aplique en la escuela.

De acuerdo con lo anterior, los datos de la entrevista aplicada a los docentes muestran que en la Institución Educativa Germán Gómez Peláez, hay una brecha entre las concepciones de enseñanza del lenguaje que poseen los docentes y las prácticas que estos realizan a diario en el aula de clases, además de que, entre uno y otro docente, hay marcadas diferencias en sus discursos respecto de la enseñanza y aprendizaje del lenguaje. Veamos lo expresado por los docentes respecto de la siguiente pregunta: ¿Coméntenos acerca del enfoque que usa para orientar el proceso de enseñanza y aprendizaje del lenguaje?:

"La enseñanza del lenguaje parte de un enfoque funcional y comunicativo, puesto que se trata de comprender la función social del lenguaje, es decir que los estudiantes comprendan la utilidad y aplicación de los contenidos lingüísticos en cualquier contexto. Además, el proceso de enseñanza y aprendizaje debe implicar la construcción de saberes desde la interacción". (Doc-1)

"Comprendiendo que el aprendizaje es personal, centrado en objetivos y que necesita una constante retroalimentación. Principalmente basado en una buena relación entre los elementos que 
participan en el proceso. Construyendo un aprendizaje significativo en los estudiantes, respondiendo y actuando ante los retos educativos del nuevo siglo". (Doc-2)

Al poner en contraste, las respuestas de los educadores vemos como el Doc-1 tiene una concepción clara del enfoque comunicativo de enseñanza, mientras que el Doc-2 divaga en sus ideas al respecto. Sin embargo, el Doc.1, dice expresiones como "aprendizaje centrado en objetivos"o "el aprendizaje personal" que dan cuenta del tradicionalismo académico en que está sumergido el educador, lo que evidencia la diferencia con la actual tendencia que sugiere trabajar en función del aprendizaje, a través de procesos, desde un enfoque sociocultural, comunicativo y participativo, que propenda por el desarrollo de competencias y no de cumplimiento de objetivos.

Por otra parte, el Doc-1, a pesar de asumir, según sus respuestas, un enfoque comunicativo y funcional, "centrando su atención en las competencias y habilidades comunicativas", al preguntarle sobre los referentes teóricos en los cuales se sustenta para orientar su práctica educativa no dio respuesta alguna, al igual que los otros tres educadores entrevistados. Esto muestra una falta de correspondencia entre saber qué se hace (la práctica), mas no desde dónde y para qué se hace (constructo teórico). La anterior consideración coincide con la idea de que el fin esencial de la educación lingüística y literaria es la mejora de las destrezas comunicativas, pero no siempre estamos de acuerdo sobre cómo contribuir de la manera más eficaz al logro de ese propósito (Lomas, 2018).

A la falta de cohesión de los educadores con respecto a sus concepciones y prácticas de enseñanza del lenguaje, se suma un discurso vago, que no precisa, al preguntarles sobre las prácticas de lectura y escritura, pues vuelven a responder generalidades, como en el siguiente caso:

"El proceso del aprendizaje del lenguaje, lo desarrollo de una manera donde tengo en cuenta a cada estudiante, sus condiciones de aprendizaje y el contexto de cada uno de ellos. Mi rol es el de crear oportunidades que le permitan desarrollar todo su potencial, capacidades y saberes demostrándoles un ambiente de confianza sumamente motivador y promover los medios necesarios para que puedan desplegar todas sus potencialidades, es decir, creo un aprendizaje significativo donde les doy la oportunidad de crear un significado nuevo al contenido relacionándolo con sus conocimientos previos". (Doc-3)

Todo esto permite comprender que aún se continúa haciendo énfasis en la enseñanza memorística y abstracta de las estructuras lingüísticas; metodología que está poco recomendada para el desarrollo de habilidades comunicativas y significativas como: lectura, escritura, oralidad y escucha). Todavía impera la idea de dictar la clase, con base en un tema específico para el cumplimiento de objetivos operacionales. Se necesita, entonces, un proceso de reflexión profundo por parte de los docentes que les permita la unificación de criterios sobre el enfoque más apropiado que deberían usar para la enseñanza de la lengua, y más concretamente para el desarrollo de habilidades de pensamiento crítico y escritura razonada. Además de eso, se debe trabajar para que haya correspondencia entre lo que se dice verbalmente sobre el proceso de aprendizaje y el desarrollo de este en el aula de clases. 


\section{Secuencias didácticas para la lectura crítica y la escritura argumentada.}

El trabajo didáctico a partir de secuencias didácticas, como se propuso en este estudio, se orientó bajo la comprensión de estas como ciclos de enseñanza y aprendizaje formados por un conjunto de actividades articuladas y orientadas a una finalidad, es decir, a la producción y comprensión de textos de prensa escrita, pretendiendo articular de forma explícita los objetivos, los contenidos y las actividades en un proyecto de trabajo interpretativo o de producción verbal, a través de una planificación minuciosa y adaptada a una situación educativa concreta y contextualizada (Dolz, J.; Noverraz, M. y Schneuwly, B. (2001). Bajo esta consideración se analiza a continuación el desarrollo de secuencias didácticas de lectura crítica y escritura argumentada.

La socialización y comprensión de los resultados de la fase anterior, respecto de la falta de unidad de criterios conceptuales y metodológicos para la enseñanza de la lectura y la escritura, permitió llegar a algunos acuerdos entre los docentes participantes respecto de un sistema de estrategias posible para motivar y orientar a los estudiantes hacia la realización de ejercicios de comprensión y producción textual a partir del uso de periódicos en el aula. Esto fue posible gracias a la realización de varios talleres de fundamentación teórica y práctica encaminados a comprender las diferencias entre los paradigmas formal y comunicativo del lenguaje, entre un enfoque estructuralista basado en la enseñanza teórica del sistema de la lengua (gramática normativa) y otro de carácter pragmático (textual y discursivo) que busca comprender cómo funciona el lenguaje en sus usos comunicativos y significativos, en situaciones de interacción social a través de diferentes tipologías textuales y géneros discursivos. La siguiente tabla describe una secuencia, no rígida, de actividades para el desarrollo de la lectura crítica y la escritura argumentada.

Tabla 2. Esquema de una secuencia didáctica.

\begin{tabular}{|c|c|}
\hline Lectura crítica & Escritura argumentada \\
\hline $\begin{array}{l}\text { Reconocimiento de los medios de comunicación y } \\
\text { su función. } \\
\text { Identificación de la relación de los medios y su } \\
\text { mensaje con el contexto. } \\
\text { Formación en criterios para seleccionar una } \\
\text { información. } \\
\text { Selección de las columnas de opinión de manera } \\
\text { anticipada por el docente. } \\
\checkmark \text { Aplicación de las técnicas de lectura y su } \\
\text { funcionalidad, (ejercicios prácticos). } \\
\checkmark \text { Selección de textos de diferentes formatos: } \\
\text { descriptivo, } \\
\text { argumentativo para analizar sus diferencias. } \\
\checkmark \text { Análisis de elementos micro y macro estructurales } \\
\text { de los artículos. } \\
\text { Identificación de tesis, argumentos y conclusión } \\
\text { de un artículo de opinión. } \\
\checkmark \text { Ejercicio de la duda frente al contenido de los } \\
\text { medios de comunicación: prensa y revista. }\end{array}$ & $\begin{array}{l}\checkmark \text { Selección de temas de interés. } \\
\text { Elaboración del plan textual. } \\
\text { Lectura en profundidad sobre temas de interés. } \\
\text { Redacción del primer borrador del artículo y } \\
\text { aplicación de distintos ejercicios: cambiar el } \\
\text { enunciado, cambiar el orden gramatical de las } \\
\text { oraciones, descomponer el sentido y orden de los } \\
\text { párrafos, definir la intención y el tono según el } \\
\text { contexto. Analizar el uso de palabras según } \\
\text { categorías: verbos, sustantivos, adjetivos para } \\
\text { tener mayor claridad de su uso. Revisión de } \\
\text { elementos microestruturales (Tejido textual), } \\
\text { atendiendo construcción cohesiva del texto para } \\
\text { lograr la coherencia (Macroestructura). Identificar } \\
\text { aspectos problemáticos de sintaxis, ortografía y } \\
\text { léxico apropiado de acuerdo con la situación de } \\
\text { comunicación y de enunciación. } \\
\text { Ejercicios de reescritura individual y grupal de los } \\
\text { textos, mediados por intervenciones magistrales } \\
\text { del docente tutor. }\end{array}$ \\
\hline
\end{tabular}


\begin{tabular}{|l|ll|}
\hline $\begin{array}{l}\text { Confrontando información teniendo en cuenta la } \\
\text { posición ideológica de las editoriales. }\end{array}$ & $\checkmark \begin{array}{l}\text { Redacción del segundo borrador del texto. } \\
\text { Retroalimentación por parte del docente a modo } \\
\text { Análisis y posición crítica del lector frente al } \\
\text { artículo de opinión. }\end{array}$ \\
$\checkmark \begin{array}{l}\text { Análisis de la situación de enunciación en los } \\
\text { textos leídos: enunciadores, voces. }\end{array}$ & $\checkmark \begin{array}{l}\text { Relectura y socialización de artículos en formato } \\
\text { digital. }\end{array}$ \\
$\checkmark \begin{array}{l}\text { Valoración de las columnas de opinión según } \\
\text { criterio propio. }\end{array}$ & \\
$\checkmark$ & final del mismo.
\end{tabular}

Fuente: Elaboración propia a partir de Tobón, Pimienta y García (2010)

Dos logros importantes hasta aquí a nivel de los docentes: uno, el estar de acuerdo en apropiar colectivamente un enfoque de enseñanza del lenguaje, venciendo así el trabajo insular y descontextualizado; y dos, el hecho de proyectar una serie de acciones didácticas un tanto diferentes de la clase tradicional del lenguaje, basada en dictar un tema, para dar paso a la práctica de enseñanza y aprendizaje mediante un proceso secuencial y sistemático, en función del desarrollo de competencias en lectura y escritura.

En cuanto a los estudiantes, lograron comprender, inicialmente, los elementos locales que constituyen los textos periodísticos (micro, macro y superestructuras - y macro-reglas), lo mismo que las diferencias y semejanzas entre artículos de opinión y el editorial. Luego, pusieron en práctica la lectura de textos, y evidenciaron, a partir de la interpretación micro y macrotextual, los estilos propios de los columnistas, lo mismo que sus tendencias ideológicas, al tiempo que se acercaron al conocimiento del periodismo como una forma global y social de comunicación que también tiene una representación ideológica, política y de poder. En este caso, fue necesario que los estudiantes entendieran que la información que leen en los medios hay que asumirla con desconfianza, es decir, que hay que analizarla en profundidad y tomar una posición crítica frente a lo leído, debido a que "los medios de comunicación juegan un papel fundamental en la conformación de cogniciones sociales del público en general" (Van Dijk, 2003: p. 232), que en ciertos casos contribuyen a crear falsos imaginarios colectivos.

Además de lo anterior, se trabajó sobre el conocimiento de la estructura formal de un artículo de opinión, es decir, que aparte de identificar aspectos como el título, el autor, el medio en el que se publica y el tipo de lenguaje, comprendieran la tesis o idea central del texto y su intención o propósito comunicativo, reconocieran también los argumentos expuestos para sustentar la idea central y relacionar entre sí, las ideas propuestas con el fin de entender de forma clara el punto de vista del autor. Para ello, se utilizaron, en lo conceptual, las macro-reglas propuestas por Van Dijk (2003), y en lo operativo, el uso de la hemeroteca, en la que los estudiantes seleccionaron y escogieron los textos (Columnas de opinión) a trabajar, de acuerdo con las actividades previamente planificadas y concertadas con el docente del grupo.

A continuación, una muestra de un ejercicio de los estudiantes que ilustra tanto la competencia de lectura crítica como la de escritura argumentada: 
LA INVASIÓN DEL ESPACIO PÚBLICO EN PUERTO LIBERTADOR

$$
\text { Por: }
$$

Sandra Patricia Díaz Rodríguez

Institución Educativa Germán Gómez

Se llama espacio público, al espacio de propiedad estatal, dominio y uso público. Es el lugar en donde cualquier persona tiene el derecho a circular en paz y armonía, donde el paso de las personas no puede ser restringido por criterios de propiedad privada y excepcionalmente por reserva gubernamental.

En Puerto libertador pareciera que este concepto de espacio público no está claro, debido a que no se cumple el concepto en su totalidad, pues no es el lugar donde cualquier persona tiene el derecho a circular en paz y armonía, donde el paso peatonal en algunos lugares es restringido por criterios de propiedad privada y excepcionalmente por reserva gubernamental.

Lo anterior es evidente, si observamos que el espacio público en nuestro municipio se encuentra convertido en espacio privado para el uso de: algunos comerciantes, algunos vendedores ambulantes, algunos moto taxistas, algunos propietarios de vehículos públicos y particulares, sin el más mínimo respeto por el peatón y por falta de reglamentación y exigencia gubernamental en el Municipio.

Pero lo más lamentable es que quienes tienen la obligación constitucional y legal de defender el espacio público para las personas (peatones), en el municipio no parecen cumplir con sus deberes y algunos comentan que están corrompidos y aceptan prebendas económicas de quienes utilizan estos espacios de dominio público para el intereses particulares.

La ley 9 de 1989 establece, que es competencia de las alcaldías locales, como dependencia de la secretaria distrital de gobierno conocer sobre la presunta invasión, el indebido uso o afectación del espacio público destinado a la satisfacción y necesidades colectivas.

Con respecto a la estadísticas, no se conoce antecedentes investigativo en este tema, lo que nos impide saber con exactitud los caso existentes y los accidentes por incumplimiento de esta norma; lo que sí se observa y no se puede esconder es que mientras transitas por las principales calles del Municipio y pasas por el frente de los negocios y sectores comerciales, notas con preocupación la invasión del espacio público por: carros, motos, bicicletas, tracto mulas descargando mercancías, exhibición de mercancía en zonas peatonales, etc.

Según el investigador en movilidad, docente Benjamín José Calle González, el peatón y la comunidad en generar no defienden el espacio público por falta de cultura en la defensa de sus derechos (Desconocimiento de la ley 9 de 1989). El anterior desconocimiento, impide las exigencias de los espacios públicos necesarios para transitar y así evitar accidentes como: ser atropellados por motos, carros, bicicletas u otros medios de transporte.

Entre los espacios públicos más violentados u ocupados en el Municipio tenemos: la plazoleta de la gran vía, la calle principal o calle del comercio, bodegas ubicadas en sectores céntricos del municipio, entre otros.

Como podemos leer, la problemática de la invasión del espacio público en Puerto Libertador es sumamente grave y no tiene dolientes: autoridades civiles y militares, sociedad civil, ONG, academia, grupo religioso, investigadores etc.

Por lo tanto, se hace necesario que las autoridades asuman su responsabilidad en la recuperación del espacio público y el libre tránsito en las calles por parte de los peatones sin que corran peligro.

Si lográramos este sagrado cumplimiento por parte de estas autoridades, se mejoraría la locomoción y la movilidad de los peatones, nos ahorraríamos una inminente tragedia anunciada, sin contar con las consecuencias que ello traería para las finanzas del Municipio (Demandas por pérdidas humanas y daños a terceros).
Título: breve, preciso y enfocado hacia el problema.

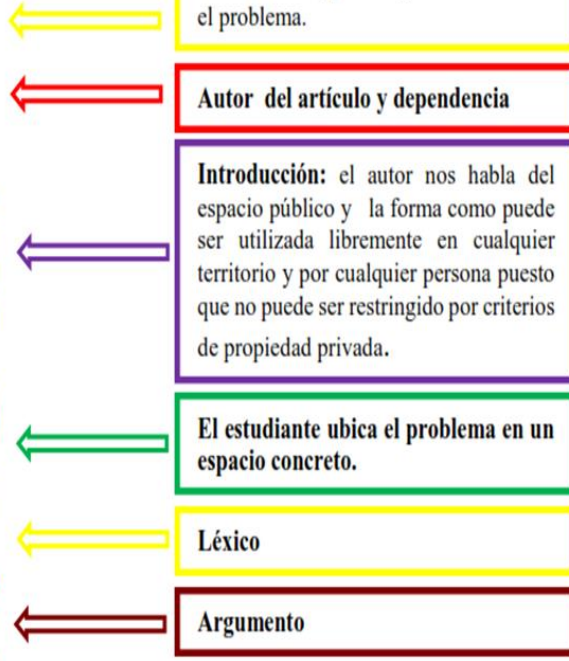

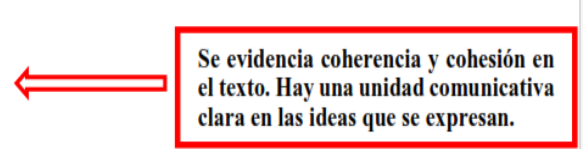

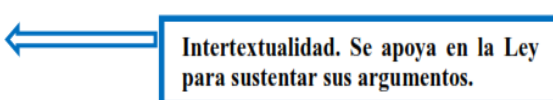
para sustentar sus argumentos.

Contextualización del problema

Crítica a las entidades responsables del control del espacio público en Puerto Libertador.

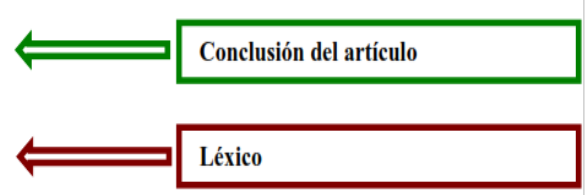


El texto anterior da cuenta de un ciclo de aprendizaje previo, mediado por lecturas de artículos de opinión de diferentes periódicos y revistas, y ejercicios de elaboración te textos con base en las secuencias anotadas anteriormente. En el caso del texto "La invasión del espacio público en puerto libertador", se nota que cumple con la estructura fundamental de este tipo de textos, puesto que se seleccionó un tema contextual, se definen unos conceptos que dan cuenta de un proceso de consulta del autor, se plantea una tesis (introducción), se plantean unos argumentos apoyados en fuentes creíbles, se intertextualiza referenciando esas fuentes, hay elemento críticos y de toma de posición, se construye una conclusión potente del texto, se observa un buen manejo de la cohesión y coherencia textuales, se apropia un léxico de buen nivel para soportar el sentido crítico de artículo. Por último, nivel pragmático, el texto es contundente en tanto relaciona la situación social vivida y reconoce el flagrante irrespeto a los derechos del peatón; una crítica que incluye aspectos extralingüísticos (irónica, tono, metáfora) que seguramente producirán diversas reacciones entre los potenciales lectores.

\section{Valoraciones y aprendizajes de los participantes en la experiencia}

Se refiere a los conceptos de los estudiantes sobre la validez de las estrategias didácticas aplicadas (secuencias didácticas), y los resultados encontrados a lo largo del proyecto. Aquí se relacionan algunas voces de los actores implicados en la investigación:

Tabla 3. Percepciones de los estudiantes.

\begin{tabular}{|c|c|}
\hline \multicolumn{2}{|c|}{ Percepciones de algunos estudiantes frente al proceso } \\
\hline Acciones & Comprensiones \\
\hline $\begin{array}{l}\text { "Trabajar con este tipo de actividades me } \\
\text { permite conocer más sobre la prensa y los } \\
\text { periódicos y también a valorar la información } \\
\text { que quieren transmitir" }\end{array}$ & $\begin{array}{l}\text { "Aprendí que mundialmente existe un programa } \\
\text { conocido por como: prensa - escuela, que busca } \\
\text { enseñar a leer a la gente para que pueda leer el } \\
\text { periódico y que ese programa en córdoba lo lidera el } \\
\text { Meridiano de Córdoba, en Puerto Libertador y } \\
\text { específicamente en la Institución Educativa Germán } \\
\text { Gómez Peláez" }\end{array}$ \\
\hline $\begin{array}{l}\text { "Considero que he adquirido varias } \\
\text { herramientas para mejorar mi comprensión de } \\
\text { lectura" }\end{array}$ & $\begin{array}{l}\text { "Entendí, que en el mundo circulan todos los días } \\
\text { centenares de columnas de opinión, escrita por los más } \\
\text { expertos profesionales en todas las disciplinas del } \\
\text { conocimiento y que cada una tiene una intención o } \\
\text { mensaje que hay que comprender" }\end{array}$ \\
\hline $\begin{array}{l}\text { "Al escribir varias columnas de opinión, se me } \\
\text { facilitó porque comprendí lo que el profesor } \\
\text { nos explicaba sobre la redacción" }\end{array}$ & $\begin{array}{l}\text { "Trabajar con este tipo de actividades me permite } \\
\text { conocer más sobre la prensa y los periódicos y también } \\
\text { a valorar la información que quieren transmitir" }\end{array}$ \\
\hline
\end{tabular}

Fuente: Elaboración propia a partir del registro de percepciones de los estudiantes. 
Comprendieron, además:

"que las salas de redacción y el trabajo diario que en ellas se realiza es concertado y en equipo, y casi que totalmente direccionado a seleccionar determinada información de acuerdo con la línea editorial del periódico o los intereses de los propietarios del mismo, por lo que se requiere de un lector con capacidad de discernimiento para leer y entender el mensaje y, a su vez, no dejarse manipular como suele suceder con los lectores pasivos". (Doc. Tutor)

En el proceso realizado se logró:

"que un alto porcentaje de los estudiantes leyeran críticamente los textos periodísticos seleccionados y produjeran sus propias columnas de opinión con claridad y consistencia argumentativa, además de haber asumido con responsabilidad el trabajo textual relacionado con la coherencia, la cohesión, el uso correcto de la gramática y la ortografía, lo mismo que aspectos discursivos como la intención comunicativa, las estrategias de enunciación y la evaluación de los textos de acuerdo con la ética y la estética de la comunicación". (Doc. Tutor)

\section{Conclusión}

Utilizar herramientas metodológicas que promuevan la lectura crítica y la escritura argumentada en la escuela, es una labor imprescindible y permanente de maestros de lengua y literatura, si se quiere superar la falta de hábito y de capacidades de comprensión y producción textual de estudiantes de educación básica y media. Para ello, habrá que pensar en la generación de una cultura de autoformación colectiva entre maestros, que les permita re-conceptualizar y re-estructurar las concepciones del lenguaje y su enseñanza, acorde con los contextos socio-históricos en que vivimos, para luego reorientar sus prácticas de enseñanza y aprendizaje con el propósito y la responsabilidad social que les compete de formar lectores críticos y escritores autónomos, tal como lo reclama el desarrollo científico y social del mundo global.

El uso del periódico en la clase de lenguaje, como un libro complementario, que ayude a aumentar los conocimientos de nuestra lengua materna, es de gran importancia, y más aún porque ofrece la posibilidad de trabajar de manera creativa y significativa haciendo uso de las Tecnologías de la información y Comunicación (TIC), que hoy día permiten una mayor interacción entre docentes y estudiantes, también, un mayor avance en la adquisición de competencias comunicativas y finalmente una mejor comprensión del mundo y adquisición de nuevos conocimientos. El trabajo en el aula a partir de secuencias didácticas no solo es una manera de superar la obsolescencia de la clase tradicional, sino que, además propicia un ejercicio pedagógico interactivo, dinámico y situado; permite también, comprender que el aprendizaje como indagación fortalece el vínculo entre saber-cultura-formación, es decir, maestros y estudiantes, juntos, a la vez que abordan desde su contexto sociocultural acciones de investigación y reflexión alrededor de temas diversos, asumiendo la lectura y la escritura como ejes estructuradores del proceso de búsqueda y de construcción intelectual y personal de los sujetos participantes. 
Teniendo en cuenta lo anterior, las instituciones educativas están llamadas a generar políticas curriculares que consideren la lectura y la escritura como elementos fundamentales para el desarrollo de competencias en todas las áreas, bajo el liderazgo del área de lengua castellana. Es decir, si la escuela como institución formadora no impulsa el desarrollo de competencias en lectura y escritura, difícilmente se podrá avanzar hacia mejores niveles de calidad. Finalmente, también se debe incluir la lectura permanente del periódico por parte de la comunidad educativa, en virtud de que el periódico es como un libro que se escribe todos los días, es una ventana de papel y virtual por la que puedes asomarte y ver todo lo que sucede. La lectura del periódico es un viaje a través del tiempo y el espacio. Por eso las personas que leen prensa están mejor informadas sobre lo que sucede en la ciudad, en el departamento, en el país y el mundo.

\section{Referencias}

Atorresi, Ana y Colaboradores (2012). Aportes para la enseñanza de la lectura. Segundo estudio regional comparativo y explicativo SERCE. Bogotá: Kimpres.

Blasco, J., Pérez, J. (2007). Metodologías de investigación en las ciencias de la actividad física y el deporte: ampliando horizontes. Universidad de Alicante: Editorial Club Universitario, p. 374.

Calsamiglia, H., y Tusón, A. (1999). Las cosas del decir. Ariel Letras

Cassany, D. (2003). Aproximaciones a la lectura crítica: teoría, ejemplos y reflexiones. Barcelona: Revista de Investigación e Innovación Educativa, (32), 113-132.

Díaz-Barriga, A. (2013). Guía para la elaboración de una secuencia didáctica. México: Universidad Nacional Autónoma de México.

Dolz, J. y Schneuwly, B. (1998). Pour un enseignement de l'oral. Paris, ESF.

Dolz, J.; Noverraz, M.; Schneuwly, B. (2001). Séquences didactiques pour l'oral et pour l'écrit: Notes méthodologiques. Bruxelles, De Boeck, vol. II, 3e/4e. En: Las secuencias didácticas de lengua: una encrucijada entre teoría y metodología. https://www.researchgate.net/publication/303357232.

Litwin, E. (1997). Las configuraciones fidácticas. Buenos Aires: Paidós.

Lomas, C. (2018) Enseñar Lengua y Literatura para aprender a comunicarse. La educación Lingüística y literaria en secundaria.

Martínez, M. (2001). Catedra UNESCO para la lectura y la escritura en América Latina. Cali: Universidad del Valle.

Ministerio de Educación Nacional (1998). Lineamientos Curriculares de Lengua Castellana. Bogotá.

Ministerio de Educación Nacional. MEN. (2012). Programa Todos a Aprender -PTA- en Colombia.

Van Dijk, T. (2003). Racismo y discurso de las élites. Barcelona: Gedisa.

Vargas, I. (2012). La entrevista en la investigación cualitativa: nuevas tendencias y retos. Centro de Investigación y Docencia en Educación, Universidad Nacional, Costa Rica.

Revista Assensus | Vol. 5 | Núm. 8 | 2020 |

https://doi.org/10.21897/assensus.2136 